\title{
Colangiopancreatografía retrógrada endoscópica (CPRE) transgástrica asistida por laparoscopia en un paciente con derivación gástrica en Y de Roux. Reporte de un caso y revisión de la literatura
}

\section{Laparoscopic-assisted transgastric retrograde endoscopic cholangiopancreatography in a patient with a Roux-en-Y gastric bypass: Case report and literature review}

Arecio Peñaloza Ramírez, ${ }^{1 *}$ Fabio Contento Anaya, ${ }^{1}$ Juan Ramírez Rueda, ${ }^{2}$ Adriana Córdoba Chamorro, ${ }^{2}$ Pedro Aponte Ordóñez. ${ }^{1}$

\footnotetext{
- Programa de Especialización en Gastroenterología y Endoscopia Digestiva, Fundación Universitaria de Ciencias de la Salud, Sociedad de Cirugía de Bogotá. Servicio de gastroenterología y endoscopia digestiva, Hospital de San José. Bogotá D. C., Colombia.

2 Programa de Especialización en Cirugía General, Fundación Universitaria de Ciencias de la Salud, Sociedad de Cirugía de Bogotá. Servicio de cirugía general, Hospital de San José. Bogotá D. C. Colombia.

^Correspondencia: apenaloza@fucsalud.edu.co.

El presente trabajo no se ha presentado en ninguna reunión de carácter académico 0 asistencial.

Fecha recibido: $16 / 03 / 18$

Fecha aceptado: 21/08/18
}

\begin{abstract}
Resumen
La obesidad es un problema de salud pública. La cirugía bariátrica juega un papel importante en el manejo de estos pacientes. Con la llegada de estas técnicas quirúrgicas, los procedimientos endoscópicos digestivos y en especial la colangiopancreatografía retrógrada endoscópica (CPRE) se convierten en un desafío constante. Se describe un caso de CPRE transgástrica asistida por laparoscopia para el manejo de cálculos de la vía biliar principal en un paciente con antecedente de derivación gástrica en Y de Roux (BPGYR).
\end{abstract}

\section{Palabras clave}

Derivación gástrica en $Y$ de Roux, colangiopancreatografía retrógrada endoscópica (CPRE) transgástrica, laparoscopia.

\begin{abstract}
Obesity is a public health problem. Bariatric surgery plays an important role in the management of these patients. With the advent of bariatric surgical techniques, endoscopic digestive procedures, especially endoscopic retrograde cholangiopancreatography (ERCP), have become constant challenges. We describe a case of laparoscopic-assisted transgastric retrograde endoscopic cholangiopancreatography (ERCP) to treat calculi in the main bile duct of a patient with a history of a Roux-en- $Y$ gastric bypass.
\end{abstract}

\section{Keywords}

Roux-en-Y gastric bypass, endoscopic retrograde endoscopic cholangiopancreatography (ERCP), laparoscopy.

\section{INTRODUCCIÓN}

La obesidad es una enfermedad creciente y de proporciones epidémicas globales que afecta a aproximadamente a 600 millones de personas según datos reportados por la Organización Mundial de la Salud (OMS) (1).

En Colombia, según datos aportados del análisis de situación de salud (ASIS) de 2016; la prevalencia para el 2010 fue un $20 \%$ mayor en comparación con el 2005, pasando de 13 a 16 casos por cada 100 personas (2).
En la actualidad, la cirugía se ha convertido en una opción mundialmente aceptada y efectiva para el control de la obesidad a largo plazo; sin embargo, con la llegada de estas técnicas quirúrgicas, los procedimientos endoscópicos digestivos se convierten en un desafío constante por el surgimiento de complicaciones propias de la cirugía $(3,4)$.

En América Latina se han publicado algunas experiencias previas de colangiopancreatografía retrógrada endoscópica (CPRE) transgástrica por vía laparoscópica $(5,6)$. El objetivo del presente trabajo es discutir el abordaje mediante 
esta técnica para el manejo de cálculos de la vía biliar principal en un paciente que consultó con antecedente de derivación gástrica en $Y$ de Roux (BPGYR).

\section{CASO CLÍNICO}

Se trata de una paciente de 70 años con antecedente de derivación gástrica en 2008 y colecistectomía laparoscópica en 2013, quien consultó por un cuadro clínico de 1 año de evolución consistente en dolor abdominal. Se documentó por colangiorresonancia la presencia de un cálculo de $5 \mathrm{~mm}$ en el extremo distal del colédoco, por lo cual se hospitalizó para la realización de CPRE transgástrica asistida por laparoscopia por el antecedente de BPGYR que altera la anatomía y el abordaje endoscópico habitual de la vía biliar.

Previo abordaje laparoscópico que permitió el ingreso del duodenoscopio al estómago, se pasó a la segunda porción duodenal donde se localizó la papila mayor de aspecto usual (Figura 1). Se canalizó la vía biliar extra e intrahepática que se observa dilatada y con un cálculo en su interior. Se realizó una esfinterotomía biliar mediante electrocirugía y se exploró la vía biliar con canastilla logrando la extracción del cálculo anotado sin complicaciones.

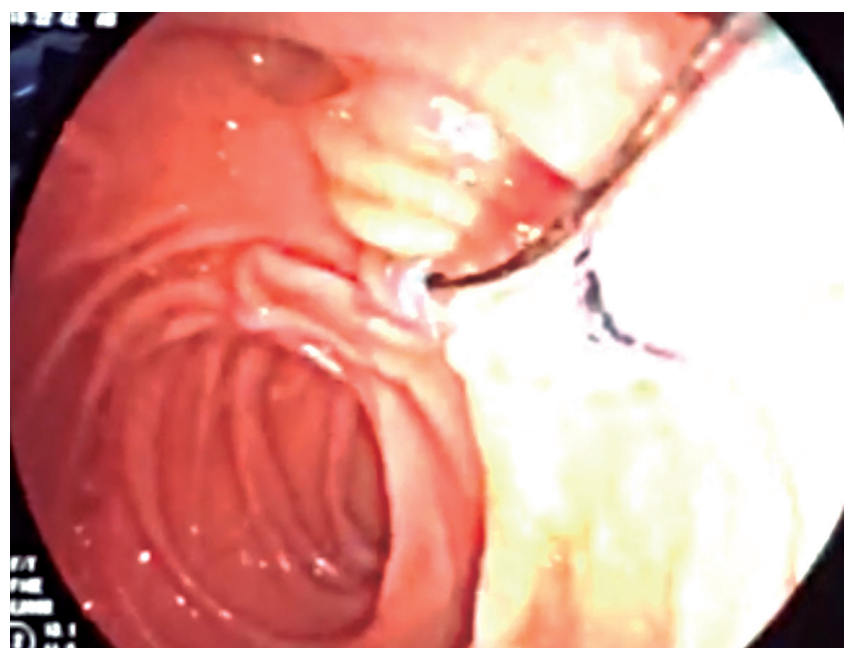

Figura 1. Enfrentamiento de la papila mayor y canalización de la vía biliar con papilotomo de arco.

A marzo de 2018 la paciente permanece asintomática digestiva.

\section{DISCUSIÓN}

La rápida pérdida de peso en los pacientes sometidos a BPGYR se ha considerado un factor de riesgo para la formación de cálculos biliares (3); la pérdida de peso postoperatorio de más del $25 \%$ del peso original se asoció con la formación de cálculos biliares sintomáticos (7) y cerca del $35 \%$ de los pacientes evolucionó con litiasis biliar en los primeros 12 meses después de la cirugía bariátrica (8).

La CPRE, en condiciones normales, se realiza con un duodenoscopio de visión lateral introducido a través de la boca hasta la segunda porción del duodeno para canalizar la papila. Es una técnica exitosa que combina la imagen endoscópica y radiológica. Sin embargo, incluso en manos expertas, es propensa a complicaciones como hemorragia, perforación, colangitis y pancreatitis (9). Por esta razón, se considera una técnica de endoscopia avanzada que requiere entrenamiento especial $(10,11)$. Teniendo en cuenta lo anterior, las dificultades y complicaciones inherentes a la CPRE aumentan cuando se realiza en anatomía alterada.

Cuando se realiza la CPRE en pacientes con anatomía alterada existen diferentes retos importantes que determinan las tasas de éxito del procedimiento como la capacidad de intubación duodenal, la capacidad de enfrentar la papila mayor, la capacidad para canalizar e intervenir la papila mayor, el éxito terapéutico, el tiempo total de la intervención y las complicaciones inherentes al procedimiento. Debido a estas dificultades, se debe realizar en centros de referencia para endoscopia avanzada con el apoyo de un equipo multidisciplinario de gastroenterólogos, radiólogos, cirujanos y anestesiólogos que facilite las tasas de éxito y atenúe el número de complicaciones (8).

No existe un protocolo sobre cómo realizar CPRE en pacientes poscirugía bariátrica. Diferentes tipos de endoscopios y técnicas endoscópicas se pueden utilizar dependiendo de la disponibilidad y la experiencia local (11).

Recientemente se han publicado recomendaciones para la realización de CPRE en presencia de anatomía alterada (12); es así como en los pacientes con anatomía Billroth II el acceso biliar es comparable cuando se usan endoscopios de visión frontal o lateral; sin embargo, estos últimos se asocian con un mayor riesgo de perforación (12). En estos casos, la papila mayor se puede alcanzar ya que el asa aferente es relativamente corta, pero los principales desafíos siguen siendo la intubación duodenal y la intervención de la vía biliar. Al respecto, una serie de casos que incluyó 713 pacientes mostró tasas de éxito de intubación duodenal del $86 \%$ y canalización de la vía biliar del $94 \%$ con una tasa de perforación global del 1,8\% (13).

En los pacientes sometidos a BPGYR, la CPRE asistida por enteroscopia (simple o de doble balón) es una de las opciones recomendadas. La técnica de la CPRE asistida por enteroscopia de doble balón consiste en avanzar a través del intestino delgado inflando y desinflando los balones hasta llegar a la papila mayor y así poder canalizar la vía biliar. Un estudio multicéntrico con 129 pacientes con BPGYR en quienes se realizaron 159 CPRE demostró acceso duodenal en un $69 \%$ con la enteroscopia de doble balón y en un $72 \%$ con la enteroscopia simple; con tasas de éxito de la CPRE del 88 \% (14). 
En cuanto a la CPRE asistida por enteroscopia simple, recientemente se publicó un metaanálisis que evaluó el éxito diagnóstico y terapéutico en pacientes con BPGYR, hepaticoyeyunostomía o cirugía de Whipple (15 ensayos clínicos, 461 pacientes); en general, el éxito de la CPRE por enteroscopia simple fue del $81 \%$, con una tasa de eventos adversos del 6,5\% (15).

Debido a que el acceso por vía oral es difícil en pacientes con derivación gástrica y a las desventajas de la CPRE asistida por enteroscopia como son la mayor duración del procedimiento, mayor dificultad para la orientación del equipo de endoscopia y menor maniobrabilidad, la disponibilidad de accesorios confiables y útiles que permitan aumentar el rendimiento diagnóstico y terapéutico del procedimiento, se ideó un método alternativo, el cual consiste en alcanzar la papila mayor a través del estómago con asistencia laparoscópica. Esta técnica fue originalmente descrita por Baron (3). La CPRE transgástrica asistida por laparoscopia ha demostrado ser superior a la CPRE asistida por enteroscopia en términos de intubación duodenal, identificación de la papila mayor, tasas de canalización biliar, éxito terapéutico y tiempo total del procedimiento; sin encontrarse diferencias estadísticamente significativas en relación con los días de hospitalización y tasas de complicaciones $(16,17)$.

Recientemente, Snauwaert ha publicado un estudio retrospectivo, multicéntrico y observacional de una cohorte de pacientes recolectados durante el período comprendido entre el 2008 y el 2014 con historia de BPGYR que presentaron patología biliar complicada (colangitis, coledocolitiasis o pancreatitis biliar) y se sometieron a CPRE transgástrica asistida por laparoscopia. Un total de 23 pacientes fueron sometidos al procedimiento, con tasas de canalización biliar del $100 \%$; sin complicaciones (sangrado, pancreatitis o perforación) y con estancia hospitalaria promedio de 2,8 días (rango: 2-4) (18).

En los últimos años, se ha utilizado otra técnica endoscópica para realizar CPRE en los pacientes con historia de BPGYR que consiste en utilizar la ultrasonografía endoscópica (USE) para obtener acceso al estómago excluido y realizar una CPRE transgástrica. Este procedimiento se realiza en dos etapas durante sesiones endoscópicas por separado: la primera etapa consiste en identificar el remanente gástrico a través de la USE y alojar una sonda de gastrostomía, la segunda etapa consiste en retirar la sonda de gastrostomía y a través de la fistula dejar una prótesis metálica que permitirá la introducción del duodenoscopio para realizar la CPRE. Un estudio publicado con 6 pacientes sometidos a este procedimiento demostró que el acceso guiado por USE del remanente gástrico excluido fue exitoso en el $100 \%$ de los casos. El tiempo medio del procedimiento fue de 81 minutos. La CPRE anterógrada se realizó con éxito en los 6 pacientes, con las dos etapas separadas por una media de 5,8 días (19).
En nuestro caso, se canalizó la vía biliar de manera exitosa, se realizó la esfinterotomía biliar y se exploró la vía biliar con canastilla, con lo que se logró la extracción del cálculo sin complicaciones. La paciente evolucionó de manera satisfactoria y fue dada de alta a las 72 horas.

En conclusión, el abordaje transgástrico por vía laparoscópica ofrece distintas ventajas como el rápido acceso al duodeno y la adecuada visualización de la papila, que permite la utilización del duodenoscopio de visión lateral, lo que aumenta el rendimiento diagnóstico o terapéutico, y finalmente, la posibilidad de un posterior acceso en caso de ser requerido. Aunque la intervención quirúrgica es necesaria, la gastrostomía por vía laparoscópica está asociada con bajas tasas de morbimortalidad. Por esta razón, la CPRE transgástrica asistida por laparoscopia es un método seguro y exitoso para el manejo de la patología biliar en pacientes con anatomía modificada luego de BPGYR.

\section{Conflictos de interés}

Ningún autor declara conflictos de interés.

\section{Fuentes de financiación}

Este trabajo no contó con financiación.

\section{REFERENCIAS}

1. Organización Mundial de la Salud. Informe sobre la situación mundial de las enfermedades no transmisibles. OMS [internet] 2017 [acceso el 15 de febrero de 2018]. Disponible en: https://www.who.int/es/news-room/factsheets/detail/obesity-and-overweight.

2. Ministerio de Salud y Protección Social. Análisis de Situación de Salud (ASIS) Colombia. MinSalud [internet] 2016 [acceso el 15 de febrero de 2018]. Disponible en: https:// www.minsalud.gov.co/sites/rid/Lists/BibliotecaDigital/ RIDE/VS/ED/PSP/asis-colombia-2016.pdf.

3. Secchi T, Falcao M, Cardoso A, Galvao M. Colangiopancreatografía endoscópica retrógrada por acceso laparoscópico transgástrico. En: Campos J (editor). Endoscopia en cirugía de la obesidad. Sao Paulo: Amolca; 2009. p. 361-7.

4. Ramírez Rueda J, Garzón J. Cirugía bariátrica en el Hospital de San José, Bogotá D. C. Experiencia y resultados. Reper Med Cir. 2010;19:187-94.

5. Branco AJ, Noda RW, Kondo W, George MA, Rangel M. Colangiopancreatografía endoscópica retrógrada transgástrica laparoscópica poscirugía bariátrica. Rev Col Bras Cir. 2008;35:445-46. https://doi.org/10.1590/S010069912008000600016.

6. Aparcero M, Pacheco J, Giannopoulos I, Izzy A, Guerere K, Díaz A. Colangiopancreatografía asistida por laparoscopia en bypass gástrico y fistulotomía pre-corte por cálculo impactado en papila. GEN. 2017;71(1):13-6. 
7. Li VK, Pulido N, Fajnwaks P, Szomstein S, Rosenthal R, Martinez-Duartez P. Predictors of gallstone formation after bariatric surgery: a multivariate analysis of risk factors comparing gastric bypass, gastric banding, and sleeve gastrectomy. Surg Endosc. 2009;23(7):1640-4. https://doi. org/10.1007/s00464-008-0204-6.

8. Moreels TG. Endoscopic retrograde cholangiopancreatography in patients with altered anatomy: How to deal with the challenges? World J Gastrointest Endosc. 2014;6(8):34551. https://doi.org/10.4253/wjge.v6.i8.345.

9. Adler D, Baron T, Davila R, Egan J, Hirota W, Leighton J, et al. ASGE guideline: the role of ERCP in diseases of the biliary tract and the pancreas. Gastrointest Endosc. 2005;62(1):18. https://doi.org/10.1016/j.gie.2005.04.015.

10. Peñaloza-Ramírez A, Leal-Buitrago C, Rodríguez-Hernández A. Adverse events of ERCP at San José Hospital of Bogotá (Colombia). Rev Esp Enferm Dig. 2009;101(12):837-49.

11. Moreels TG. ERCP in the patient with surgically altered anatomy. Curr Gastroenterol Rep. 2013;15(9):343. https:// doi.org/10.1007/s11894-013-0343-3.

12. Liao WC, Angsuwatcharakon $P$, Isayama $H$, Dhir V, Devereaux $\mathrm{B}$, Khor CJ, et al. International consensus recommendations for difficult biliary access. Gastrointest Endosc. 2017;85(2):295304. https://doi.org/10.1016/j.gie.2016.09.037.

13. Bove V, Tringali A, Familiari P, Gigante G, Boškoski I, Perri $\mathrm{V}$, et al. ERCP in patients with prior Billroth II gastrectomy: report of 30 years' experience. Endoscopy. 2015;47(7):6116. https://doi.org/10.1055/s-0034-1391567.
14. Iorgulescu A, Turcu F, Iordache N. ERCP after bariatric surgery-literature review and case report. J Med Life. 2014;7(3):339-42.

15. Inamdar S, Slattery E, Sejpal DV, Miller LS, Pleskow DK, Berzin TM, et al. Systematic review and meta-analysis of single-balloon enteroscopy-assisted ERCP in patients with surgically altered GI anatomy. Gastrointest Endosc. 2015;82(1):919. https://doi.org/10.1016/j.gie.2015.02.013.

16. Schreiner MA, Chang L, Gluck M, Irani S, Gan SI, Brandabur JJ, et al. Laparoscopy-assisted versus balloon enteroscopyassisted ERCP in bariatric post-Roux-en-Y gastric bypass patients. Gastrointest Endosc. 2012;75(4):748-56. https:// doi.org/10.1016/j.gie.2011.11.019.

17. Bertin PM, Singh K, Arregui ME. Laparoscopic transgastric endoscopic retrograde cholangiopancreatography (ERCP) after gastric bypass: case series and a description of technique. Surg Endosc. 2011;25(8):2592-6. https://doi. org/10.1007/s00464-011-1593-5.

18. Snauwaert C, Laukens P, Dillemans B, Himpens J, De Looze D, DeprezPH, etal.Laparoscopy-assisted transgastricendoscopic retrograde cholangiopancreatography in bariatric Roux-en-Y gastric bypass patients. Endosc Int Open. 2015;3(5):E45863. https://doi.org/10.1055/s-0034-1392108.

19. Kedia P, Kumta NA, Widmer J, Sundararajan S, Cerefice M, Gaidhane M, et al. Endoscopic ultrasound-directed transgastric ERCP (EDGE) for Roux-en-Y anatomy: a novel technique. Endoscopy. 2015;47(2):159-63. https://doi. org/10.1055/s-0034-1390771. 